\title{
Magnetoelastic Energy Harvesting: Modeling and Experiments
}

\author{
Daniele Davino, Alessandro Giustiniani and Ciro Visone
}

Additional information is available at the end of the chapter

http://dx.doi.org/10.5772/50892

\section{Introduction}

Magnetoelastic materials belong to the wide category of smart materials because of their capability of coupling mechanical quantities (force, strain) to magnetic ones (field, induction) and viceversa. Recently, they have received a lot of interest for actuating and sensing purposes. Moreover, in the general framework of recovering some environmental energy, this kind of smart materials have been considered to recover the mechanical energy of vibrations [51].

Indeed, by employing the inverse magnetostriction or Villari Effect [26], it is possible to scavenge the vibration energy by means of the induced magnetization change in the material to generate electrical power. This opens the possibility to have a regenerative source of electrical power, especially useful in harsh environments. For this reason, this kind of technology can result of great interest in several application fields, such as health monitoring of civil infrastructure (bridges, buildings), automotive and biomedical tasks. Magnetostrictive alloys (Terfenol, Galfenol, Metglas) are actually the most known and employed magneto-elastic materials in this kind of applications. Nevertheless, they have interesting properties like high energy densities, high bandwidth, absence of depolarization phenomena that make them complementary to the piezoelectrics.

Energy harvesting techniques from vibrations have a promising future in civil engineering, where a strong need of structural monitoring of the health of ageing bridges and structures is occurring [17]. Indeed, for all civil infrastructure in general, it is possible to infer the structural health by measuring accelerations and resonant frequencies [21, 31]. Usually, the resonant frequencies are measured in suitable places along the structure by using the vibrations induced by wind or traffic $[4,27,30]$. Moreover, the sensors can detect other local environment parameters as well, namely temperature, wind speed, humidity, etc. Once the data are measured, among all the possible transmission techniques, the wireless method is 
undoubtedly the more effective because, for example, it reduces costs with respect to periodic human intervention and it improves reliability with respect to wired solutions. The use of sensors together with wireless transmission results in the so called wireless sensors networks (WSN) [7]. Of course, in the same line of reasoning, the source of electrical power for the WSN should be self-contained too. The easiest choice could be then the use of batteries but, due to the limited life-span of them, an increment of the sensors maintenance whole costs should be considered, with the aggravation of workers safety concerns because the sensors can be located in inconvenient places along the bridge. Then, smart renewable energy methods should be used instead. Solar and thermal harvesting have been proposed [43] but these solutions can be both costly and bulky. Nevertheless, bridges as many other civil infrastructure vibrate because of the wind action and of traffic loadings or, when presents, for trains traffic loadings. The possibility to convert this ambient mechanical energy, otherwise wasted, into electrical energy is very attractive in those applications [36, 39]. So, together with measurements purposes, vibrations can be harvested to feed the sensors.

It is worth that this type of conversion could be performed by means of linear electromagnetic generators too $[20,47]$. In those devices, a proof mass oscillates with the structure, making a permanent magnet move linearly in a coil. This solution is undoubtedly well assessed and reliable. But, usually, this type of device has a narrow bandwidth that can be broaden at the cost of a very sophisticate mechanical construction. Another conversion technique, as already introduced, can make use of smart materials instead. These devices can be in principle less bulky and with higher reliability, because of their simpler mechanical design and construction.

Another field of application for energy harvesting from vibration is the automotive one. In fact, on one hand, the society is looking forward for vehicles more and more efficient. On the other hand, moving vehicle are site of vibrations of two different kinds. The first is due to the internal combustion engine operations and so, whenever the engine is on, a source of vibration is present. These vibrations are usually damped by means of engine rubber supports or even more complicated ways, while they could be used to recover some more energy. Obviously, the vibrations level is higher in big vehicles like trucks or tractors. A second source of vibrations can be referred as external and it is intrinsically related to the vehicle movement and interaction with the roads irregularities. It is apparent that both of them can be exploited to scavenge electrical power, instead of wasting the energy in rubber damping or shock absorbing. Also in this case, the harvested power can be used to recharge the vehicle battery or for feeding monitoring sensor nodes. A typical example is a tyre pressure sensor, [46]. In this case, the knowledge of the tyre health and pressure status allows to reduce the fuel consumption and to limit accidents. Another example of application on vehicles is the mechanical energy harvesting on the suspension system. Noting that the electrical energy transfer on the load (e.g. the battery) is strictly related to the mechanical source damping, this kind of solution can be conceived also for obtaining shock absorption, [54]. This additional feature is very attractive for enhancing the comfort of the passengers, particularly for workers (e.g. on trucks or tractors).

The harvesting from vibrations can be also considered in flying vehicles. In this case the vibrations are induced on the wings by the movement in the air flow. In [2], a sheet of 
piezoelectric material on the wings of an unmanned plane is applied making a harvester in a sort of unimorph cantilever arrangement.

Another potential huge field of application for vibrations harvesting is the biomedical one [32]. In this case the source of vibrations is the human gait (walking and running) and it could be used to power devices aimed to monitor human health conditions, [18], out of personal multimedia readers, smartphone, etc. In this framework of human activities induced vibrations, even the movement of the heart muscle has been conceived as a possible energy source to feed internal biomedical devices as pacemakers, [23].

Aim of this chapter is to discuss the main achievements and the open challenges in the field of vibrations energy harvesters based on magnetostrictive materials. This is a very attractive field involving the modeling of active materials that, with their complex behavior, are the link between the mechanical and the electrical worlds and so represent the path by which a smart electromechanical conversion can take place.

Different modeling strategies will be considered, ranging from the basic linear one [22, 45] allowing to understand the device working principles to the more accurate nonlinear approaches $[10,50]$, outlining their impact on the practical design of the harvester. To this aim, some detail on the experimental setup for material characterization, [1,11], the modeling of the mechanical source and its coupling to the active material will be presented.

Finally, many open problems will be also reviewed, such as the power conversion stage requirements, the main arrangements (bulk or cantilevers) of magnetostrictive harvesters in connection to the different fields of applications.

The chapter is structured as follows:

- Section 2 focuses the modeling of a magnetostrictive energy harvester discussing the basic components of the device, with particular emphasis on the active material. To this aim both linear and nonlinear modeling approaches are considered, highlighting their impact on the harvester performances prediction.

- Section 3 presents some experimental results necessary to the characterization of the material parameters and to evidence the basic phenomena involved.

- Section 4 is focused on some open problems related to the magnetostrictive harvesting, on new materials and on modeling challenges.

\section{Magnetoelastic energy harvester modeling}

This section aims to introduce different basic approaches that can be considered when a magneto-elastic energy harvester is modeled. Particular emphasis will be devoted to the material modeling, starting with the classic description under the general hypothesis of linearity (in analogy with the typical modeling of piezoelectric materials), as well as with more sophisticated approaches, taking into account the material nonlinearities and rate-independent hysteresis. Then, the whole device is modeled from the magneto-mechanical point of view. In the scientific literature, usually, a semicoupled modeling approach is adopted: the mechanical stimulus is ideal and only its effect on the magnetic 
characteristic is considered. On the other hand, a fully coupled approach can be considered and, in this case, the influence of the material response on the mechanical side can be taken into account too, if the vibration source is not ideal.

This analysis will be done employing an analogy with a circuital description by means of the two-port circuits formalism. As interesting application, the capabilities of the harvesting process to damp the vibrations of the mechanical source will be discussed.

All the analysis will be performed under the following fundamental assumptions:

(a) All the field are coaxial and directed along the magneto-elastic material axis,

(b) the length of the structure along the field axis is much larger than the other dimensions,

(c) the vibrations frequencies are much lower than the mechanical resonance of the structure, i.e. sound propagation can be neglected,

(d) the electric load is a lumped element, i.e. electromagnetic field propagation is neglected.

The first one is necessary to have, together with an isotropic material behavior, a scalar description of the constitutive relationships. The second one allows, for instance, to have the long solenoid hypothesis for the coil and to treat the mechanical stress as uniform along the material. The third and the fourth assumptions allow to neglect any propagation effect into the device.

\subsection{Material modeling - constitutive relationships}

In the hypothesis of monodimensional operating regime (all the fields and the mechanical input along the same direction), the magneto-elastic characteristics can be written as:

$$
\left\{\begin{array}{l}
S=S(H, T) \\
B=B(H, T)
\end{array}\right.
$$

where $S$ is the strain, $H$ is the applied magnetic field, $T$ is the compressive stress and $B$ is the magnetic induction. Typical behaviors of those characteristics are shown in Fig. 1 and the following general properties can be inferred:

- $S(H)$ and $B(H)$ are non-linear with non-local memory, i.e. hysteresis,

- $S(H)$ and $B(H)$ show saturation when $|H| \rightarrow \infty$,

- $S$ and $B$ are even and odd function of $H$, respectively,

- $S(H)$ : there is an optimum $T_{0}$ that makes $\Delta S$ largest at $H_{\max }$

- $S(H)$ : if $T>T_{0}$ then the $S(H)$ cycles drop down,

- $S(H)$ : the $S(H)$ cycles are self-similar with respect to the stress,

- $B(H)$ : if the compressive $T$ increases then the $B(H)$ cycles drop down (the material becomes magnetically harder under increasing constant stress). 

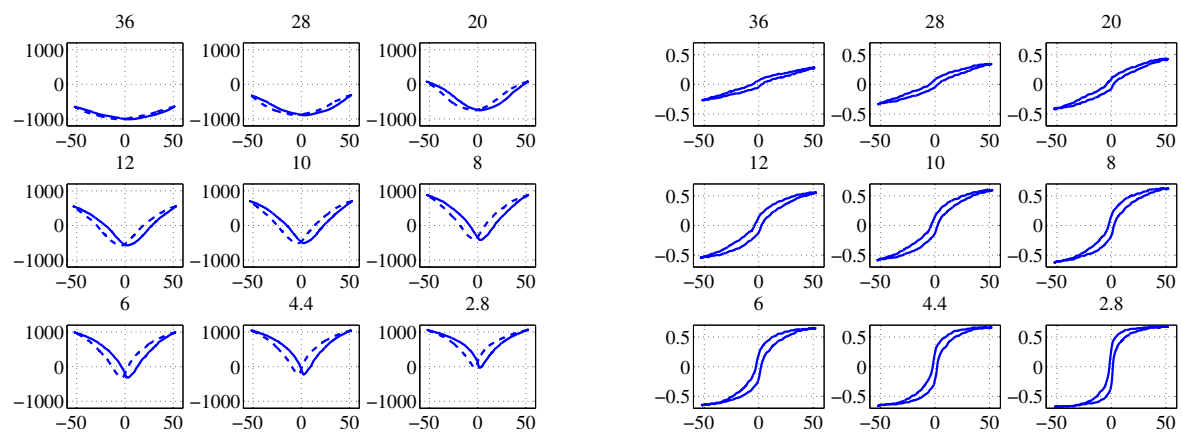

(a) Magnetostrictive cycles ( $S$ in ppm vs $H$ in $\mathrm{kA} / \mathrm{m})$.

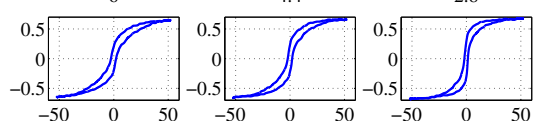

(b) Magnetic cycles ( $B$ in Tesla vs $H$ in $\mathrm{kA} / \mathrm{m}$ ).

Figure 1. Typical magnetostrictive characteristics (Terfenol-D) at different constant stresses (the titles are $|T|[\mathrm{MPa}])$.

The eqs. (1) can be obtained by suitable derivatives of the Gibbs free energy expression:

$$
G(T, H)=\frac{T^{2}}{2 E}+\frac{\mu_{0}}{2} H^{2}+\Psi(T, H) \rightarrow\left\{\begin{array}{l}
S=\left.\frac{\partial G}{\partial T}\right|_{H} \\
B=\left.\frac{\partial G}{\partial H}\right|_{T}
\end{array}\right.
$$

where the first and second terms are pure linear elastic and magnetic energies, respectively, and the third one is the magneto-elastic energy. $E$ is the Young modulus and $\mu_{0}$ is the vacuum magnetic permeability. It is worth noting that $\left.\right|_{H, T}$ means that the derivatives are made at constant $H$ and $T$, respectively.

The simplest way to model the magnetoelastic materials behaviour is the linear one. In that case the Gibbs free energy expression reads:

$$
G(T, H)=\frac{T^{2}}{2 E}+\frac{\mu_{0}}{2} H^{2}+\frac{\mu_{0}}{2} \chi H^{2}+d T H \rightarrow\left\{\begin{array}{l}
S=d H+\frac{T}{E} \\
B=\mu H+d T
\end{array}\right.
$$

where $\mu=\mu_{0}(1+\chi)=\mu_{0} \mu_{r}$ and $d$ is the, so called, piezo-magnetic coefficient. It is worth noting that in this case it is assumed that any transformation taking place in the material is lossless. This assumption, from the thermodynamic viewpoint, leads to the following constraint [48]:

$$
\left.\frac{\partial S}{\partial H}\right|_{T}=\left.\frac{\partial B}{\partial T}\right|_{H}
$$

It can be noted that the eqs. (3) are the magnetic counterpart of the piezoelectric ones. Obviously, in a B-H plane (or in a S-H) at a fixed stresses $\mathrm{T}$ (or at a fixed magnetic fields $\mathrm{H})$, the linear modeling predicts parallel lines. This behavior is far from the actual material response because magnetoelastic materials show saturation phenomena and also a magnetic hardening, as stated above. So, this approach can fail in predictions when large variations of 

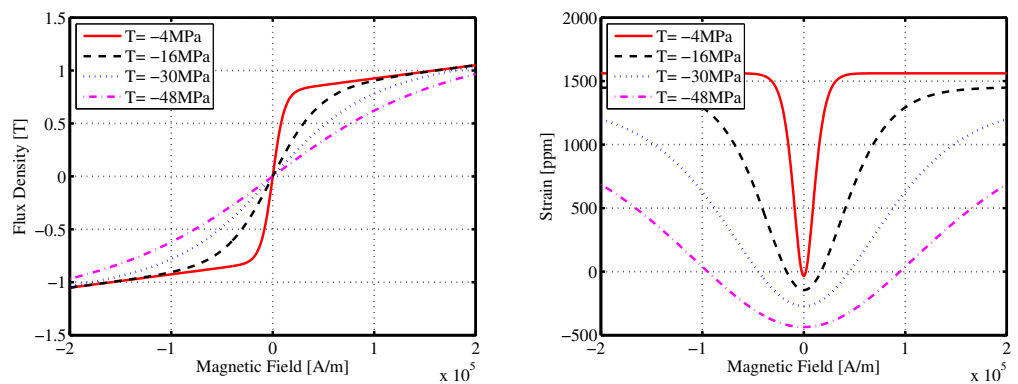

Figure 2. Nonlinear magnetostrictive characteristics obtained from eqs. (6). The values $M_{S}=0.8 \mathrm{~T}$, $\gamma=-347 \mathrm{~T}, E=110 \mathrm{GPa}$ match the typical behavior of Terfenol.

the inputs are considered but can be fruitfully used if the inputs have small variations around a magneto-mechanical working point.

A more advanced model, with the only further hypothesis to neglect the hysteresis, is represented by the following magnetic characteristic:

$$
G(T, H)=\frac{T^{2}}{2 E}+\frac{\mu_{0}}{2} H^{2}+f(T) \chi\left(\frac{H}{f(T)}\right) \rightarrow\left\{\begin{array}{l}
S=\left(\frac{\partial G}{\partial T}\right)_{H}=\frac{T}{E}-f^{\prime}(T)\left[z \chi^{\prime}(z)-\chi(z)\right] \\
B=\left(\frac{\partial G}{\partial H}\right)_{T}=\mu_{0} H+\chi^{\prime}(z)
\end{array}\right.
$$

where $z=H / f(T)$ and $f(\cdot)$ and $\chi(\cdot)$ are suitable one-variable functions and can be chosen in order to model the physical behavior of the material, as saturation effect and stress dependence [19]. Indeed, if $\chi^{\prime}(z)=M_{S} \tanh (z)$ [3] and $f(T)=\frac{T}{\gamma}$ then the magnetic characteristic can be well approximated. In this case, $\chi(z)=M_{s} \ln (\cosh z)$ and eqs. (5) become:

$$
\left\{\begin{array}{l}
S=\frac{T}{E}-\frac{M_{S}}{\gamma}[z \tanh (z)-\ln (\cosh z)] \\
B=\mu_{0} H+M_{S} \tanh \left(\gamma \frac{H}{T}\right)
\end{array}\right.
$$

where $M_{S}$ is the magnetic polarization saturation and $\gamma$ is a parameter modifying the approach to saturation which should be properly identified, as reported in [10]. As can be noted, it shows saturation by means of hyperbolic tangent and the magnetic hardening by means of the $z$ dependence for compressive stresses.

Finally, considering that also hysteresis is shown by magnetoelastic materials another generalization can be made. A phenomenological approach for including hysteresis is to introduce a Prandtl-Ishilinskii operator [24]:

$$
\pi[x]=\int_{0}^{+\infty} \xi(r) \mathcal{P}_{r}[x] d r,
$$

where $\mathcal{P}_{r}$ is a Play operator of threshold $r$ and $\xi(r)$ is a weight function that can be identified from experimental data. It is constructed by a linear superposition of Play operators, but it 
cannot model saturation phenomena. In order to circumvent this limitation and to model the magneto-mechanical coupling taking place in the magnetoelastic material, the above operator can be generalized. In particular, it can be written:

$$
B=G(\pi[H], T),
$$

with

$$
G(x, T)=\mu_{0} x+M_{s} \tanh \left(\gamma \frac{x}{T}\right) .
$$

The function $\mathrm{G}$ is the same considered in the memoryless modeling approach and allows to take into account saturation.

However, it is worth to note that when the hysteresis is introduced the method adopted for the memoryless case basing on the thermodynamic constraint (eq. (4)) cannot be considered because the process is not lossless. Moreover, this approach models only one between magnetostrictive and magnetic characteristics. This can be still valid if the vibration source can be considered ideal (semi-coupled approach). On the other hand, the problem of a fully coupled approach with hysteresis is still open.

\subsection{Device modeling}

A magnetoelastic harvester can be arranged in different configurations. They can be summarized in two main categories:

- Direct force harvesters also known as force-driven.

- Inertial harvesters also known as velocity-driven.

All the harvesters where the force source is in mechanical direct contact to the active material, in the so called longitudinal mode, belong to the first group. A representative device of such a harvester is shown in Fig. 3 (a). In this case, usually, the material is fabricated in the form of rods, disks or cymbals.

Instead, the second group exploits the inertial forces of a proof mass oscillations induced by a vibrating bond. Typical example is a cantilever, where a thin sheet of the active material is bonded on a elastic structure and bounded on one side, while on the other there is a mass free to vibrate, as sketched in Fig. 3 (b). The thin sheet of active material undergoes heavy stress variations when the mass vibrate. Conceptually, the energy conversion follows the same path of the force driven harvester. But, this configuration has a resonant-like mechanical behavior with a relatively small bandwidth and a resonant frequency related to the mass (the larger is the mass, the lower is the frequency) [44]. The first group has, instead, a larger bandwidth from the vibration frequencies point of view but a lower peak specific power. In the following we will refer to a generic harvester with particular reference to the force-driven.

Despite of the arrangements, the main elements of a magnetostrictive harvester are:

- the active material,

- the magnetic circuit,

- the coil. 


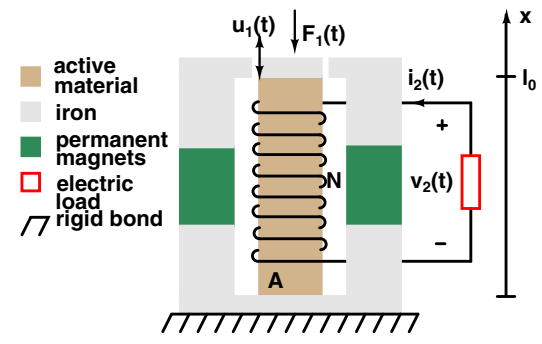

(a) Force-driven.

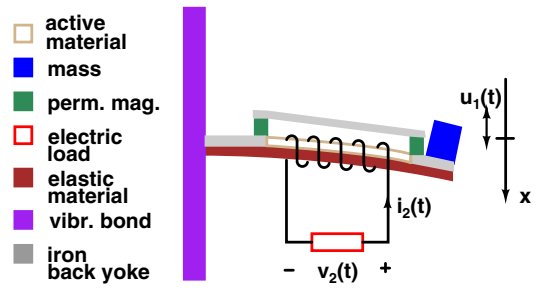

(b) Velocity-driven.

Figure 3. Magnetostrictive energy harvester concept devices.

Let us consider the sketch of a magnetostrictive harvester under an external ideal time-variable force $F_{1}(t)$, as sketched in Fig. 3 (a). The active material of such a force-driven harvesters has a rod shape. In order to achieve an easier modeling and design of the device, the rod should be a cylinder with the length $l_{0}$ far larger than the section diameter. Indeed, in this case, two hypothesis can be made: the stress and the magnetic field lines are uniform along the rod leading to, eventually to 1-D problems along the radial direction [15]. The section area $A$ should be chosen such that the available force variation gives the best stress variation for harvesting (see section 3.3). The magnetic circuit is needed to provide the magnetic bias to the active material and suitable permanent magnets can be used then. But, it is worth noting that the magnets cannot be coaxial to the active material because, usually, they are sintered and cannot stand the time-variable mechanical stresses. The N-turns coil is wound around the active material where $\mathrm{N}$ is chosen in order to achieve the best compromise between available voltage and current over the electric load. Finally, it should be noted that the modern electronic allows the construction of suitable energy conversion circuits that helps to match the electric impedance seen by the harvester itself to the best value, improving conversion efficiency then $[8,35]$.

Let us recall the eqs. (3):

$$
\left\{\begin{array}{l}
S=d H+\frac{T}{E} \\
B=\mu H+d T
\end{array}\right.
$$

Starting from these relationships, a general model of the energy harvester of Fig. 3 can be obtained. The procedure is based on the analogy of the whole system with a two-port: the first port with mechanical variables and the second with electrical variables. Then, a two-port circuit model of the magneto-elastic device can be obtained by exploiting the following analogy: the input force $F_{1}$ corresponds to a primary two-port voltage $\left(F_{1} \Leftrightarrow v_{1}\right)$; while the rod tip velocity $u_{1}=d x / d t$ corresponds to a primary current $\left(u_{1}=l_{0}(d S / d t) \Leftrightarrow i_{1}\right)$. Instead, the current $i_{2}$ and voltage $v_{2}$ on the actual magnetostrictive rod coil represent the current and voltage on the second port.

It is worth noting that such an approach exploits all the power of the lumped circuit theory, making very easy to treat different cases. Moreover, the two-port equivalent model can be solved by means of standard circuit simulators [10]. 


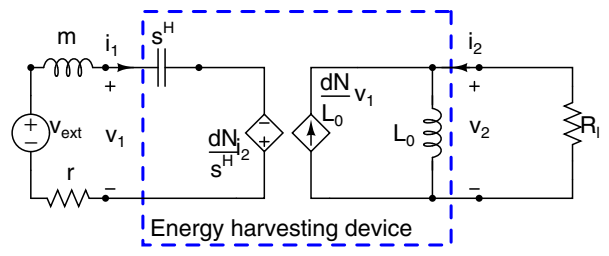

(a) Linear modeling and external elements.

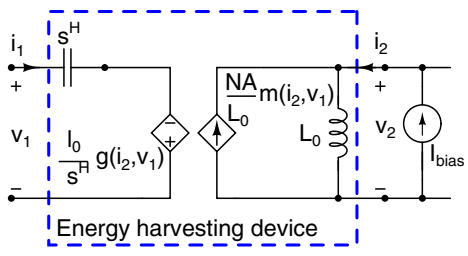

(b) Non-linear modeling.

Figure 4. Two-port equivalent circuits. (a) the part inside the dashed box implements the eqs. (12). (b) The part inside the dashed box implements the eqs. (15).

In order to do so, the local quantities of eqs. (10) have to be related to the measurables one, i.e. $F_{1}, u_{1}, v_{2}$ and $i_{2}$, by using the hypothesis already made: $H=N i_{2} / l_{0}$ (long solenoid), $\Phi_{2}=N A B, x=l_{0} S$, and $F_{1}=A T$ (an applied uniform in the space force on the material cross section $A$ is assumed), where $\Phi_{2}$ is the magnetic flux and $x$ is deformation.

So the eqs (10) can be recasted as follows:

$$
\left\{\begin{array} { l } 
{ x = \frac { l _ { 0 } } { E A } F _ { 1 } + d N i _ { 2 } } \\
{ \frac { \Phi _ { 2 } } { N A } = \frac { d } { A } F _ { 1 } + \mu \frac { N i _ { 2 } } { l _ { 0 } } . }
\end{array} \rightarrow \left\{\begin{array}{ll}
x & ={ }^{H} F_{1}+d N i_{2} \\
\Phi_{2} & =d N F_{1}+L_{0} i_{2} .
\end{array}\right.\right.
$$

where $L_{0}=\mu \frac{N^{2} A}{l_{0}}$ and $s^{H}=\frac{l_{0}}{E A}$ have been defined, the latter being the mechanical compliance at constant magnetic field $\mathrm{H}$.

By exploiting the analogies $F_{1} \Leftrightarrow v_{1}$ e $d x / d t \Leftrightarrow i_{1}$, the following relationships can be derived then:

$$
\begin{cases}\frac{1}{s^{H}} \int_{0}^{t} i_{1} d \tau & =v_{1}+\frac{d N}{s^{H}} i_{2} \\ \frac{1}{L_{0}} \int_{0}^{t} v_{2} d \tau & =\frac{d N}{L_{0}} v_{1}+i_{2} .\end{cases}
$$

These equations are implemented in the equivalent circuit of Fig. 4 (a). The first one can be interpreted as a voltage balance at the primary port: the primary voltage $v_{1}$ is equal to the voltage on a capacitor of value $s^{H}$ minus a voltage generator controlled by the secondary current $i_{2}$. Furthermore, the second of eqs. (12) can be interpreted as a current balance at the secondary port: the secondary current $i_{2}$ is equal to the current in an inductor of value $L_{0}$ minus a current generator controlled by the primary voltage $v_{1}$. For example, if the harvester undergoes a vibrating force generator with a certain mass $m$ and it is connected to a resistor $R_{l}$ then, as represented in Fig. 4 (a), this can be simply solved by connecting the series of a voltage generator and a inductor ${ }^{1}$ to the first port and, of course, the resistor to the second port.

The proposed methodology can be also employed to derive a two-port representation in the memoryless non-linear case, (5). By The eqs. (6) can be recasted as follows:

\footnotetext{
${ }^{1}$ The equation of a rigid mass is $F=m d^{2} x / d t^{2} \leftrightarrow v=m d i / d t$ that is the inductor characteristic. A linear elastic effect is equivalent to a capacitor: $F=k x \leftrightarrow v=k \int i d t$ (with the capacitance equal to $1 / k$ ) and, finally, a viscous friction is represented by a resistor: $F=r d x / d t \leftrightarrow v=r i$.
} 


$$
\left\{\begin{array}{l}
x=\frac{l_{0}}{S A} F_{1}+g\left(i_{2}, F_{1}\right) l \\
\Phi_{2}=\mu_{0} \frac{N^{2} A}{l} i_{2}+m\left(i_{2}, F_{1}\right) N A .
\end{array}\right.
$$

where

$$
\left\{\begin{array}{l}
g\left(i_{2}, F_{1}\right)=-\frac{M_{s}}{\gamma}\left[\left(\frac{\gamma N A}{l} \frac{i_{2}}{F_{1}}\right) \tanh \left(\frac{\gamma N A}{l} \frac{i_{2}}{F_{1}}\right)-\ln \left(\cosh \left(\frac{\gamma N A}{l} \frac{i_{2}}{F_{1}}\right)\right)\right] \\
m\left(i_{2}, F_{1}\right)=M_{s} \tanh \left(\frac{\gamma N A}{l} \frac{i_{2}}{F_{1}}\right) .
\end{array}\right.
$$

Assuming the same previous analogies $i_{1}=d x / d t$ and $v_{2}=d \Phi_{2} / d t$, the following system describing the nonlinear dynamic two-port can be derived:

$$
\left\{\begin{array}{l}
\frac{1}{s^{H}} \int_{0}^{t} i_{1} d \tau=v_{1}+\frac{l_{0}}{s^{H}} g\left(i_{2}, v_{1}\right) \\
\frac{1}{L_{0}} \int_{0}^{t} v_{2} d \tau=i_{2}+\frac{N A}{L_{0}} m\left(i_{2}, v_{1}\right),
\end{array}\right.
$$

and the two-port is formally unchanged, apart from the controlled generators and a DC current generator, as shown in Fig. 4 (b). The generator implements the effect of a magnetic bias over the magnetostrictive characteristics. It does not produce any dissipated power into the electric load and, therefore, does not contribute to the energy balance of the two-port.

Finally, the two-port modeling approach makes easier to consider different types of vibration sources, either force or velocity driven [8]. Indeed, the simplest model of non ideal mechanical source can be defined as:

$$
\left\{\begin{aligned}
l_{0} d S / d t & =u_{1}(t)-\frac{A}{\eta} T(t) \text { for } \eta>0 ; \\
A T(t) & =F_{1}(t)-\eta l_{0} \dot{S} \text { for } \eta<+\infty .
\end{aligned}\right.
$$

In the former case, $u_{1}$ is an impressed velocity (velocity-driven source), while in the latter $F_{1}$ is an impressed force (force-driven source). These two elements can be added to the two-port circuit as the classical current and voltage non ideal generators, respectively. The first equation leads to the ideal velocity-driven source when the mechanical resistance $\eta \rightarrow \infty$, while $\eta=0$ describes a ideal stress-driven mechanical source in the latter.

\subsection{Numerical results}

In this section the different aforementioned approaches for describing the material behavior are compared, highlighting the main features and limitations. To this aim, it is useful considering a semi-coupled problem where the mechanical stress is imposed. In this case, in fact, only the effect of the mechanical source on the harvesting performances can be considered and evaluated. In the following examples, a cylindrical active material sample with length $l_{0}=0.1 \mathrm{~m}$ and area $A=\pi \mathrm{cm}^{2}$ wounded by a coil with $N=100$ turns is considered. In Fig. 5 , the harvested power on a resistor $R=10 \Omega$ is reported, for a mechanical sinusoidal stress $T=T_{0}+T_{m} \cos (2 \pi f t)$ with $T_{0}=-27.4 \mathrm{MPa}$ and $T_{m}=13.9 \mathrm{MPa}$, at different frequencies. 




Figure 5. Harvested Power at different magnetic biases. It has been considered $\mu_{r}=12, E=30 \mathrm{GPa}$, $d=2 \cdot 10^{-8} \mathrm{~m} / \mathrm{A}$ in eqs. (12), while $M_{s}=0.8 \mathrm{~T}, \gamma=-347 \mathrm{~T}, E=110 \mathrm{GPa}$ in eqs. (6).

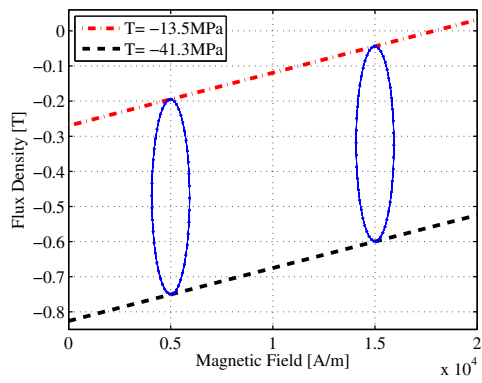

(a) Linear Modeling.



(b) Nonlinear Memoryless Modeling.

Figure 6. Harvesting periodic trajectories - varying external magnetic bias.

A first important difference between the linear and the nonlinear memoryless approach can be noted. In fact, in the linear case the harvested power is independent on the magnetic bias, while in the nonlinear case this strongly influences the harvester performances. This phenomenon can be easily understood referring to Fig. 6 where the periodic working trajectories are reported in the $\mathrm{B}-\mathrm{H}$ plane, for a fixed frequency $f=168.5 \mathrm{~Hz}$. It is worth to note that the area of this loops is directly proportional to the harvested energy, i.e. the averaged harvested power [9]. In the linear case (Fig. 6 (a)), the described loops are equal independently on the magnetic bias. Instead, in the non-linear case (Fig. 6 (b)), the loops area changes on this parameter. In the same figures the static characteristics of the two modeling approaches are reported. As it can be seen, these loops are within the static characteristics: in the linear case the distance is equal (parallel lines), in the nonlinear one this relative distance depends on the magnetic bias level. To support this interpretation, another test has been performed comparing again the linear and the nonlinear memoryless models, but varying the mechanical prestress level, keeping the magnetic bias constant, see Fig. 7. In this case $T_{0}=-20.45 \mathrm{MPa}$ or $T_{0}=-34.35 \mathrm{MPa}$ have been considered, while the stress amplitude $T_{m}=6.95 \mathrm{MPa}$ is fixed. Again the harvested energies in the linear case are the same, while in the nonlinear case are strongly influenced by the prestress level. To explain this other 


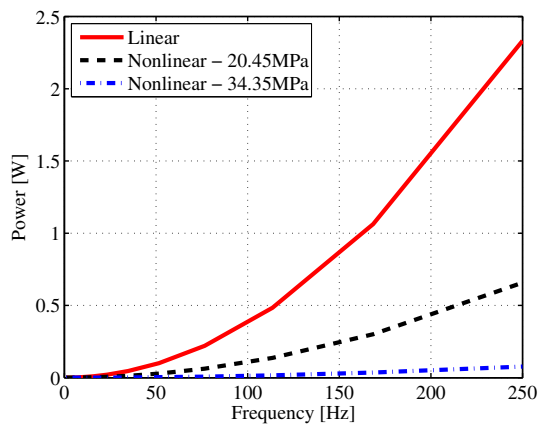

Figure 7. Harvested Power at different mechanical prestresses. The models parameters are the same of Fig. 5.

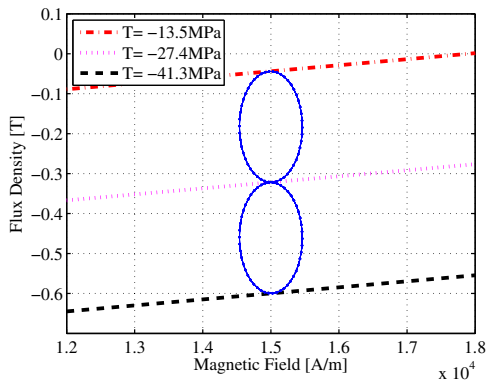

(a) Linear Modeling.

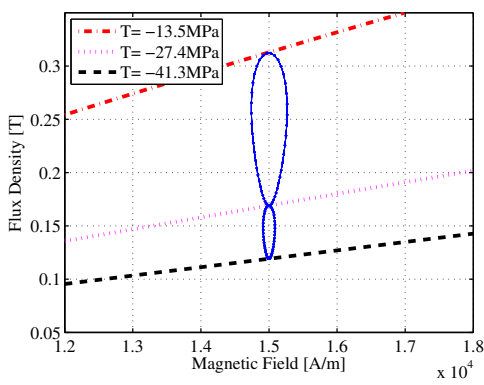

(b) Nonlinear Memoryless Modeling.

Figure 8. Harvesting periodic trajectories - varying mechanical prestress. The models parameters are the same of Fig. 5.

behavior, let us consider the Figs. 8 with loops in the B-H plane. In Fig. 8 (a), the linear static characteristics are also reported and as it can be noted the relative distance is still constant while in the nonlinear case it is not. So the described loops by the linear model are equal (same power level) independently on the prestress level. So summarizing, the linear model cannot take into account the magnetic bias and the mechanical prestress in the description of the harvester performances.

In Fig. 9, a comparison between the memoryless non-linear and hysteretic models is reported. In Fig. 9 (a), the static characteristics of the two models for two different mechanical stresses are drawn. For a mechanical input with a prestress $T_{0}=-27.4 \mathrm{MPa}$ and amplitude $T_{m}=13.9 \mathrm{MPa}$, the harvested powers on a resistor $R=10 \Omega$ are reported in Fig. 9 (b). In this case, as additional phenomenon related to the hysteresis, it can be noted that the harvested power levels changes with the initial state (in this case, the demagnetized initial state and the saturation). This behavior can be also analyzed on the B-H plane, as reported in Figs. 10 (a) and (b). While in the memoryless nonlinear case the periodic loops are within the static characteristics, when the hysteresis is considered the periodic trajectories are within the 


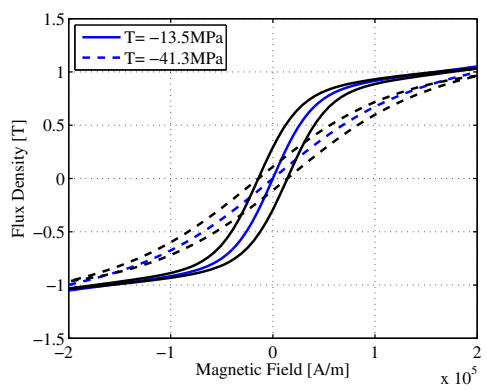

(a) Static Characteristics.

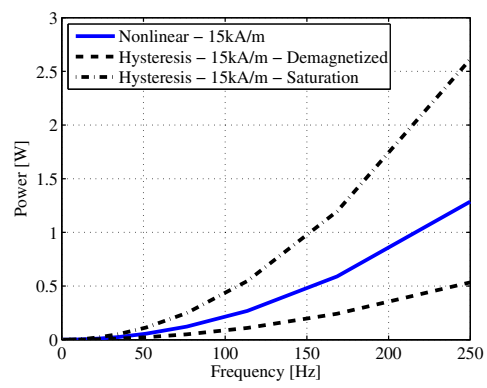

(b) Average Power.

Figure 9. Comparison between Nonlinear Memoryless and Hysteretic modeling.

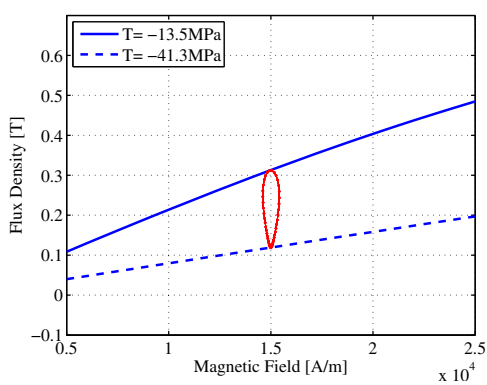

(a) Non-linear Memoryless model.

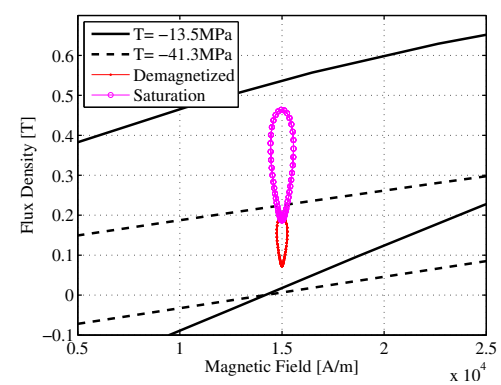

(b) Hysteretic model.

Figure 10. Harvesting periodic trajectories: comparison between non-linear and hysteretic models, starting from different initial internal states.

hysteresis loops but their position and area are strongly influenced by the initial state of the hysteresis model.

In order to analyze the effect of the harvesting process on the mechanical side, a fully coupled problem is also addressed. In this case, the first port of the equivalent two-port model is closed with a lumped mass $m=0.5 \mathrm{~kg}$ and a viscous friction $r=500 \mathrm{Ns} / \mathrm{m}$. In Fig. 11, the free response of the model with an initial velocity $v=0.1 \mathrm{~m} / \mathrm{s}$ is shown, for the linear and the nonlinear memoryless models. As it can be noted, the external magnetic bias strongly influences also the strain time evolution (see Fig. 11 (a)) as done on the electrical side (see Fig. 11 (b)). The linear modeling approach cannot take into account this dependence, however it can be considered for a preliminary analysis of the magneto-elastic harvesting device.

\section{Experimental characterization and results}

The good design of a magnetoelastic energy harvester needs the characterization of the raw active materials, by knowing their magnetomechanical characteristics. In order to do so, a rather complex experimental setup is needed because both magnetic and mechanical 


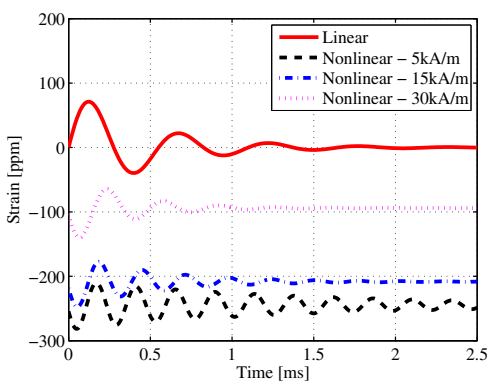

(a) Strain

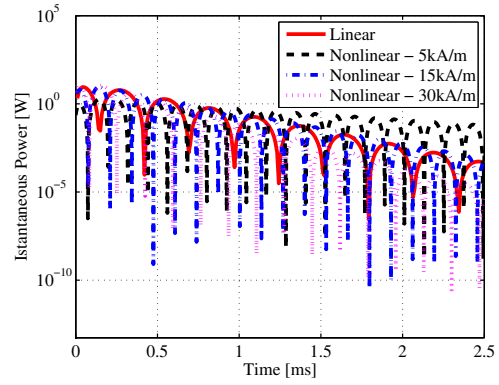

(b) Instantaneous power.

Figure 11. Impulse response - Comparison

variables must be measured and/or enforced at the same time. Morever, the constitutive relationships of magnetostrictive materials, like Terfenol-D or Galfenol, show nonlinear and rate-independent memory effect (hysteresis) and the mechanical $(S, T)$ and magnetic variables $(H, B)$ are cross-coupled [19]. The cross-coupling terms are usually named piezo-magnetic coefficients and, in the energy harvesting framework, a deep knowledge of them is crucial because they are related to the material ability to convert energy [19]. For example, the accurate knowledge or modeling of their behavior, with respect to the magnetic field and prestress biases, can be useful to choose the working point $\left(H_{0}, T_{0}\right)$ that guarantees the best performance in a energy harvesting device [10] or in other applications like a sensor. Now, in the case of characteristics without hysteresis, the piezo-magnetic parameters have to satisfy the thermodynamic constraint of eq. (4).

Several papers have tackled the experimental problem of measuring the piezo-magnetic coefficients of magnetostrictive material, as well other parameters as mechanical compliance, Young modulus, etc. This study is mandatory to explore the performance of new magnetostrictive compounds [5, 6, 37]. In a early paper, the problem of hysteresis of those coefficients started to be considered [34]. Also the variability of the piezo-magnetic parameter with the applied prestress has been considered [40], leading to the conclusion that magnetic and mechanical bias must be chosen with attention in order to get the best performance [49]. This result have been confirmed by a later paper on non-linear modeling [10].

The magnetostriction and the magnetic characteristics have to be measured in different stress and magnetic field conditions in order to explore the previous conjecture.

In order to measure the magnetostrictive characteristics, a combination of different standard and custom instruments have to be used, as sketched in Fig. 12. The mechanical compressive load is applied through a computer-controlled test-machine while the magnetic field is imposed through an electromagnet and measured by using a digital gaussmeter. The material magnetic induction is obtained after a numerical time-integration of a voltage, measured over a pick-up coil with 500 turns. The demagnetizing effect must be taken into account. The strain is directly measured with a strain gauges bridge, configured to have temperature self-compensation, while the applied stress is measured by a load cell. 


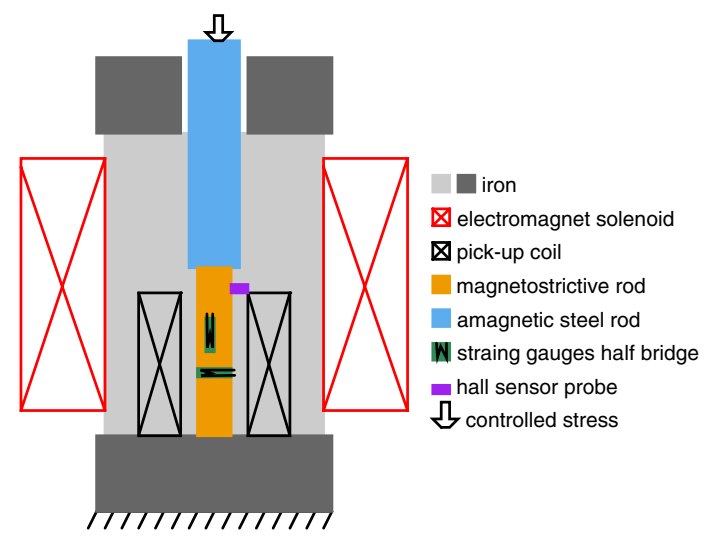

Figure 12. Sketch of a possible setup to measure the magnetostrictive characteristics.

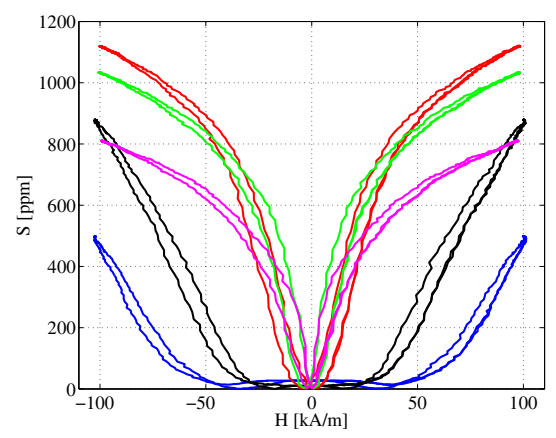

(a) Strain vs magnetic field.

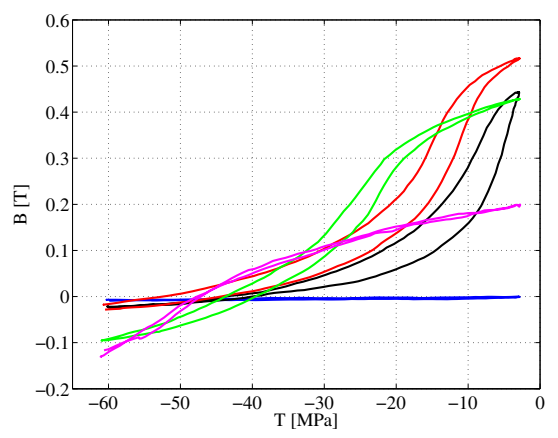

(b) Magnetic induction vs stress.

Figure 13. Magnetostrictive cycles. (a) legend: $4 \mathrm{MPa}$-fuchsia, $6 \mathrm{MPa}$-green, $12 \mathrm{MPa}$-red, $40 \mathrm{MPa}$-black, $60 \mathrm{MPa}-$ blue. (b) legend: $0 \mathrm{kA} / \mathrm{m}$-blue, $10 \mathrm{kA} / \mathrm{m}$-black, $20 \mathrm{kA} / \mathrm{m}$-red, $40 \mathrm{kA} / \mathrm{m}$-green, $100 \mathrm{kA} / \mathrm{m}$-fuchsia.

\subsection{Magnetostrictive characteristics}

The magnetostrictive characteristics $S(H)$ under variable magnetic field and constant compressive stress are shown in Fig. 13 (a). The magnetostrictive characteristics does not include the elastic effect, i.e. the mechanical strain induced by the constant stress. It is worth noting that the dependence from the applied constant stress is somewhat complex. Indeed, the cycles change in shape and maximum amplitude with the stress.

Finally, the Fig. 13 (b) shows the magnetic induction induced by a cycled stress. These measurements are very important to infer the harvesting potentialities of the material. Indeed, the larger is the $\Delta B$ at a certain $\Delta T$ the better would be the energy conversion. The measurements show wider cycles, i.e. more hysteresis, at low-intermediate magnetic field. Moreover, the maximum $\Delta B$ is reached at intermediate field too. 


\subsection{Piezo-magnetic coefficients}

The definition of the piezo-magnetic parameters considered here are the following [12, 34]:

$$
\left\{\begin{array}{l}
d_{33}=\left.\frac{\partial S}{\partial H}\right|_{T} \\
d_{33}^{*}=\left.\frac{\partial B}{\partial T}\right|_{H}
\end{array}\right.
$$

As stated in eq. (4), they are equal if the hypothesis of lossless material is considered while, in general, they are not. These parameters are usually considered constant in datasheets and in linear models of the material [34]. Such an approximation holds if the material is employed in actuators where the mechanical prestress is higher than the external stress applied to the device. On the other hand, the approximation cannot be applied if general dynamic conditions are considered, as the case of energy harvesting applications.

Indeed, the piezo-magnetic parameters are nonlinear function of the considered $(H, T)$ couple. Moreover, they show hysteresis. Nevertheless, the piezo-magnetic parameters can be obtained by a numerical derivative of the experimental hysteresis loops. The two branches of the loops are considered as up and down curves in the following.

In order to compare the two parameters, they have been sampled in a same matrix of $(H, T)$ points. The corresponding surfaces are shown in Figs. 14,15. It is apparent that the orders of magnitude and the shapes are the same, but the parameters are not exactly equal for each $(H, T)$ couple. The maximum for each set of curves (up and down) moves at higher $H$ field for higher $T$ and this confirms nonlinear models results in literature (see [8], Fig. 3 or [48]). Nevertheless, for all of them, the maximum values are achieved at relatively low stress and magnetic field, that are the ranges $T \in(-10,0) \mathrm{MPa}$ and $H \in(10,20) \mathrm{kA} / \mathrm{m}$. Those values are useful to choose the best magnetic bias and mechanical prestress.

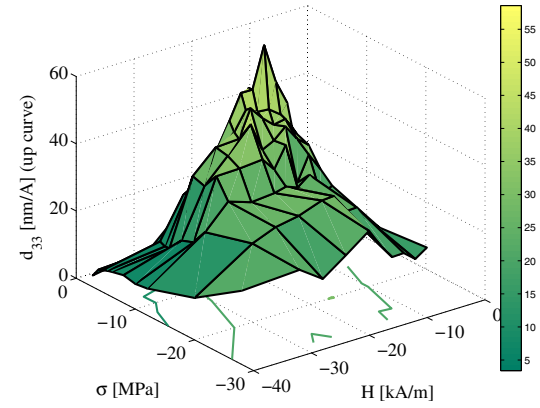

(a) $d_{33}$

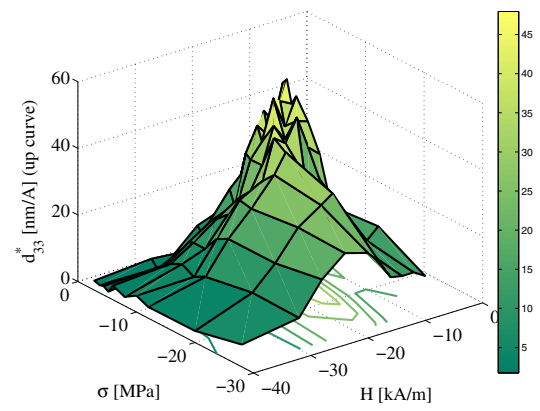

(b) $d_{33}^{*}$.

Figure 14. The piezo-magnetic parameters (up curves) sampled at different magnetic field and stress.

\subsection{Harvesting results - Terfenol}

Let us consider now the specific powers generated by a laboratory harvesting device with a Terfenol-D rod $18 \mathrm{~mm}$ long and with $9 \mathrm{~mm}^{2}$ square section. The harvester has a pick-up coil 


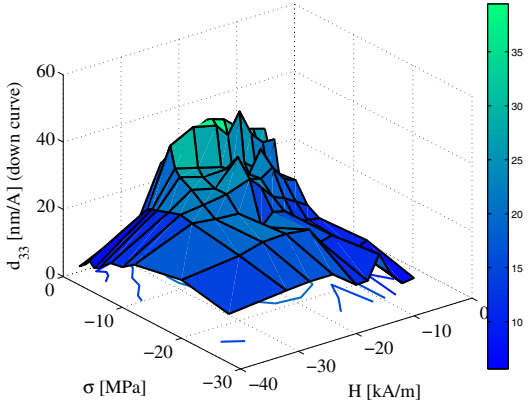

(a) $d_{33}$

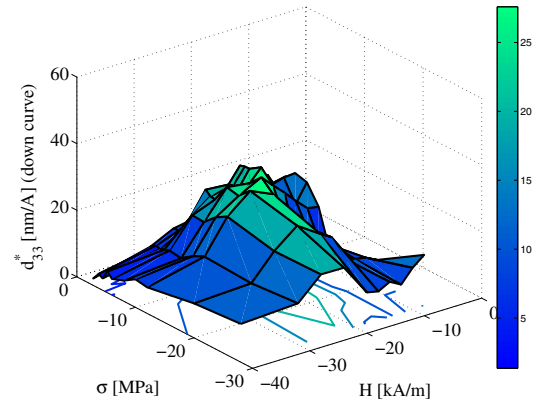

(b) $d_{33}^{*}$.

Figure 15. The piezo-magnetic parameters parameters (down curves) sampled at different magnetic field and stress.
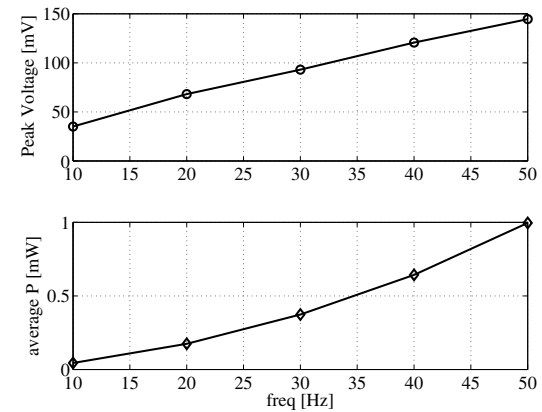

(a) Peak voltage and average power with $H_{0}=$ $18 \mathrm{kA} / \mathrm{m}, \Delta \mathrm{F}=300 \mathrm{~N}$.

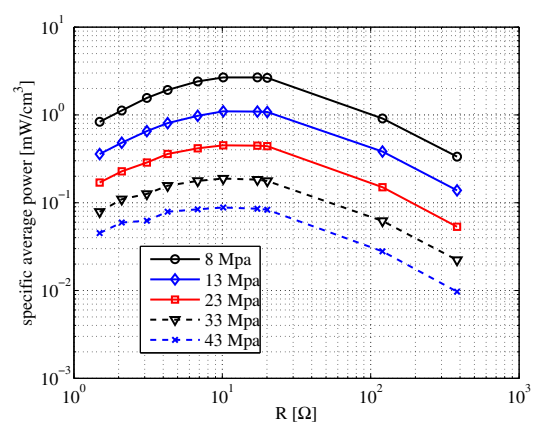

(b) Specific average power at $f=50 \mathrm{~Hz}, H_{0}=$ $4.8 \mathrm{kA} / \mathrm{m}, \Delta \mathrm{F}=310 \mathrm{~N}$ at different pre-stresses.

Figure 16. Harvesting results of a force-driven device with a terfenol rod.

with 800 turns. The Fig. 16 (a) shows the peak voltage and average power with vibration frequency and $H_{0}=18 \mathrm{kA} / \mathrm{m}, \Delta \mathrm{F}=300 \mathrm{~N}$, corresponding to $\Delta T=33.3 \mathrm{MPa}$. It is worth noting that, at $50 \mathrm{~Hz}$, a relevant specific power of $6.17 \mathrm{~mW} / \mathrm{cm}^{3}$ is reached, confirming the potentialities of vibration harvesting when high frequencies are concerned. The Fig. 16 (b) shows the average power at $50 \mathrm{~Hz}$ by varying the resistance load, at different prestresses. It is apparent that the choices of the correct prestress and resistance are crucial to harvest the maximum power.

\subsection{Harvesting results - Galfenol}

Let us consider now the specific powers generated by a laboratory harvesting device with a Galfenol rod $30 \mathrm{~mm}$ long and with $5 \mathrm{~mm}$ diameter. The Fig. 17 shows the average power densities (left) and the peak to peak voltage with a 3000 turns pick-up coil (parasitic resistance of $1 \mathrm{k} \Omega$ ) and $1 \mathrm{k} \Omega$ termination resistor. The compressive stress variation is $30 \mathrm{MPa}$ and 



Figure 17. Average power densities (left) and peak to peak voltage (right) at $f=0.35 \mathrm{~Hz}$, with a $\Delta T=30 \mathrm{MPa}$ stress variation.

$f=0.35 \mathrm{~Hz}$, a very low frequency that could be found in structural vibrations of civil constructions. It is worth noting that the peaks are reached at relatively low magnetic field bias, confirming one of the advantages to use Galfenol for harvesting applications. Indeed, those magnetic biases could be easily obtained with a permanent magnet, in a engineered harvesting device. The peak specific power is about $1 \mu \mathrm{W} / \mathrm{cm}^{3}$, reached at $10 \mathrm{kA} / \mathrm{m}$ magnetic bias and $16 \mathrm{MPa}$ prestress. Those values are reasonable and in agreement with literature values [33]. The Fig. 18 (a) shows the average power densities with respect to different termination resistors and with a 800 turns coil (parasitic resistance of $19 \Omega$ ). Moreover, the resistor value near the parasitic coil resistance gives the maximum power. The Fig. 18 (b) shows the average power density behavior with respect to the stress variation frequency with $20 \Omega$ resistance, $15 \mathrm{kA} / \mathrm{m}$ magnetic bias and with a $1-80 \mathrm{MPa}$ stress variation. As expected, the power increases with frequency.

\subsection{Harvesting loops behavior in the B-H plane}

It is a fundamental result the fact that magnetic loops in the B-H plane represent losses if the loops are passed through counterclockwise. On the other hand, if passed clockwise, those loops area represent generated energy. Then, it is apparent that a good design of the device should aim to enlarge as much as possible that area. Now, as it was shown in the previous sections, magnetic bias, prestress and other parameters can be used to do so because if in steady-state vibrations the power is increased then the loop area in the B-H plane is increased too. The Fig. 19 (a) shows the magnetic characteristics at 1.58 and $48.2 \mathrm{MPa}$ constant stresses. The blue lines within the cycles represent the loops due to harvesting tests at different magnetic bias $(8.7,14.8$ and $23 \mathrm{kA} / \mathrm{m}), 1 \mathrm{M} \Omega$ resistance and $f=0.8 \mathrm{~Hz}$. It is noticeable that the loops are contained within the magnetic characteristics, as it was theoretically foreseen in [9]. Moreover, this is still true if different resistors are employed, as shown in Fig. 19 (b). The loops are wider for smaller resistor because a larger current circulates in the pick-up 


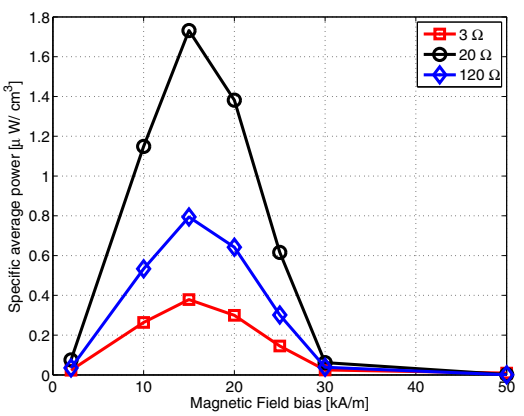

(a) Average power densities at $f=0.35 \mathrm{~Hz}$, with a $1-80 \mathrm{MPa}$ stress variation.



(b) Average power densities at $15 \mathrm{kA} / \mathrm{m}$ magnetic bias, with a $1-80 \mathrm{MPa}$ stress variation, $20 \Omega$ resistance.

Figure 18. Harvesting results of a force-driven device with a galfenol rod.

coil and a magnetic field variation is introduced then. This result is important because it is evident that the effort to improve the material harvesting behavior should concentrate on the static magnetic characteristics at different stresses, trying to improve as much as possible the available area among limit cycles at different stresses.

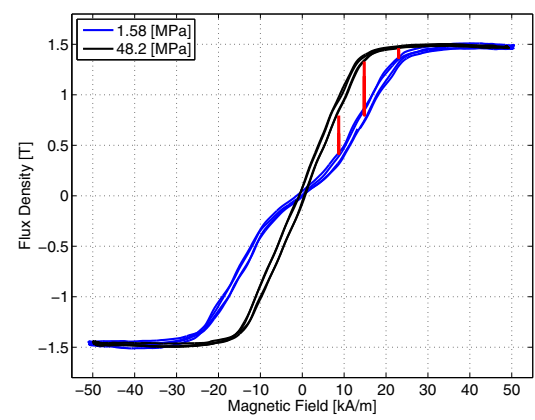

(a)

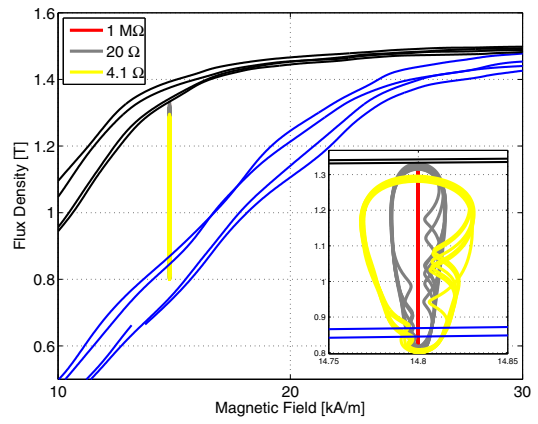

(b)

Figure 19. (a) Magnetic characteristics with converted energy loops at different magnetic bias (8.7, 14.8 and $23 \mathrm{kA} / \mathrm{m}$ ), $1 \mathrm{M} \Omega$ resistance and $f=0.8 \mathrm{~Hz}$. (b) Converted energy loops zoom with different resistance termination at $14.8 \mathrm{kA} / \mathrm{m}$ magnetic bias.

\section{Conclusions and open problems}

\section{Materials}

It is evident that the research on magnetoelastic material synthesis can give a strong advancement to the energy harvesting applications. Indeed, the research should focus to maximize the piezo-magnetic coefficients. This, as explained in section 3.5, would means to have more space for harvesting loops within static limit cycles. In other words, to have a larger $\Delta B$ for a certain $\Delta T$. 
Another topic about materials synthesis concerns the mechanical impedance matching of the harvesters. Indeed, materials like terfenol or galfenol are quite rigid with a mechanical behavior near the one of bulk iron. In that case, the ideal vibrations have high stresses and low strains, in the $0-1000 \mathrm{ppm}$ range. If a softer magnetoelastic materials would exist, it would be possible to have vibrations harvesting with lower stresses and higher strains, in the $0.1-1 \%$ range, with a rubber-like behavior.

Recently the use of another magnetoelastic material (Metglass) has been proposed for energy harvesting [45]. The main advantage respect to the others magnetostrictive material is that it can be laminated achieving a higher harvester compactness. The material is a Fe-based amorphous ribbons with excellent magnetic softness and elastic response and it is cheaper than Fe-Ga, Fe-Tb-Dy alloys. A recent new application design reached $20 \mu \mathrm{W} / \mathrm{cm}^{3}$ at $100 \mathrm{~Hz}$ [53].

\section{Models}

The further steps in the material modeling should aim to include the hysteresis in both characteristics in a thermodynamic compatible way. Indeed, up to now, this step is made only on one of the two characteristics when the other one is not relevant for the specific applications. This is valid when the vibration source can be considered almost ideal.

Another topic in the analysis of a harvester, based on magnetoelastic materials, is the eddy currents effect due to the mechanical stress variations, that can have a detrimental effect on the harvester performances [51]. This phenomenon is present when the material has a finite electric resistivity, that is the case of magnetostrictive materials as Terfenol and Galfenol. The effect can be more harmful on the latter because of the high relative permeability. From the modeling point of view, the eddy currents problem can be formulated, as for standard magnetic materials, starting from Maxwell's equations in the magneto-quasistatic limit. The main difference is that the magnetic characteristics depends on the applied stress. Then, the stress acts as the forcing term in the magneto-mechanical problem [14, 15].

\section{Damping}

An effective energy harvesting from vibrations results in an amplitude damping on the mechanical source, no matter what the harvesting method is. In other terms, though the principal aim of an harvesting device is to convert the available mechanical energy into electrical energy, at the same time a kind of passive mechanical damping occurs. The passive damping by smart materials has been generally addressed in [41]. Recent contributions deepen this damping effect for Piezo [28, 29] and Magnetostrictive materials [10, 13]. The latter presents a preliminary analysis showing the effects of the energy harvesting on mechanical damping which requires further effort. As already mentioned, the passive damping could be exploited in several application fields, for example, in automotive applications where the vibrations reduction is a concern and energy harvesting can help to improve the overall efficiency. Moreover, this concept is even more attractive if compared to the classical active mechanical damping, where an additional properly controlled actuator is needed with a consequent increase of cost, system complexity and use of energy. 


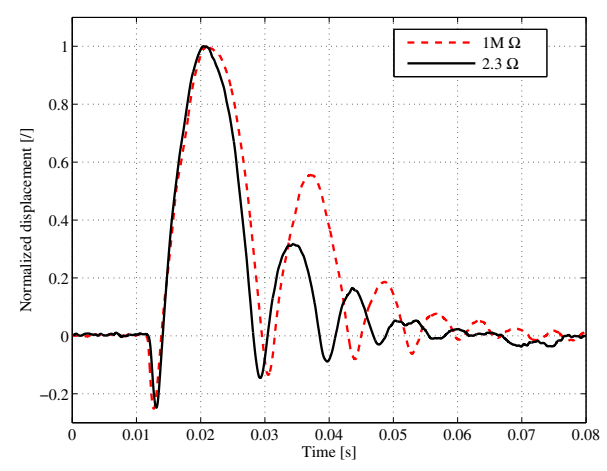

(a) Displacement of a $7.5 \mathrm{Kg}$ mass.
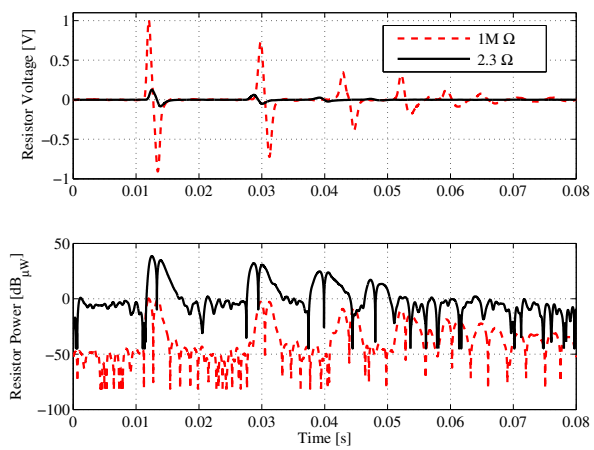

(b) Measured resistors voltage (up) and instantaneous dissipated power (down).

Figure 20. Damping effect of the vibration harvesting. Two different resistors case are reported with a $7 \mathrm{kA} / \mathrm{m}$ magnetic bias. The power $p(t)=v^{2}(t) / R$ is represented with $10 \log _{10}\left(\frac{p(t)}{1 \mu \mathrm{W}}\right)$.

In Fig. 20 (a) the normalized displacement comparison between two different resistive loads is reported (2.3 $\Omega$ - solid line, $1 \mathrm{M} \Omega$-dashed line). The Fig. 20 (b) shows the corresponding resistor voltage (up) and the instantaneous dissipated electric power (down). The mass is in mechanical contact with the active material and a controlled initial velocity is applied to them. The active material has suitable bias conditions (magnetic bias of $7 \mathrm{kA} / \mathrm{m}$ and a mechanical prestress of $2.9 \mathrm{MPa}$ ). The damping with the $1 \mathrm{M} \Omega$ resistor is due only to mechanical friction, while with the $2.3 \Omega$ resistor there is a faster damping of the oscillation, both in amplitude and in time, because of the energy harvesting. Indeed, in this case a far larger power is extracted. Finally, the voltage is higher with the $1 \mathrm{M} \Omega$ resistor as expected (a sort of open circuit condition).

\section{Power conversion circuits}

A magnetoelastic energy harvester is an unregulated AC power source. So it can not be directly interfaced to common electronic loads that need a regulated DC supply. Then, a power conversion stage must be a part of the harvester to overcome this issue. In particular, this stage should accomplish to two main functions:

- an efficient rectification;

- the regulation and level shifting of the output voltage.

These tasks can be afforded in different ways that strongly depend on the available AC power from the harvester and on the specific field of application. In fact, magnetoelastic energy harvesters output powers can range from milliwatt to watt levels depending on the mechanical source characteristics and its coupling with the active material. Moreover, also the dimensions of the magnetoelastic material influence the obtainable power, but in many applications they are limited by compactness requirements.

The choice of the topology and circuit implementation of the power conversion stage is based also on the following criteria, [42]: 
- efficiency. The power stage for its operation must consume less power as possible. This is a strict requirement in energy havesting application due to the limited generated power.

- stand-alone operation. The mechanical source is strongly time varying: if there are vibrations there is electrical power output. The power stage should also contain an independent start-up circuit.

- circuit complexity. This influences the choice of the control strategies and their implementation for the power stage.

- adaptivity. The power stage must work with a wide swing of electric inputs (due to the mechanical source) and of electric output (due to the load requests), guaranteeing the maximum power transfer to the electrical load.

The possible solutions to the above requirements and criteria can be identified in two different approaches, according to what has been done for piezoelectrics or other mature harvesting technologies, like electromagnetic and electrostatic generators:

- Single stage power conversion. In this case the rectification and the DC voltage regulation are made in a single step. To this approach belong solutions like the Direct AC-DC Switch-Mode Converters (e. g. single inductor with split capacitor, single inductor with secondary-side switches, dual-boost converters), [16]. Also specific solutions for a particular harvesting technology are possible. For the piezoelectrics, due to their intrinsic capacitive behavior, switched inductor converters (e.g. the synchronized switch harvesting on inductor (SSHI) and its generalizations) have been proposed [25, 52]. In the case of magnetoleastic harvester similar solutions could be conceived by considering their inductive nature, by duality.

- Double stage power conversion. In this case the rectification and the voltage regulation are separated. The rectification can be made with classical solutions as diodes full bridges or with the so called active diodes. The second stage instead should regulate the output voltage and its shifting. Due to the relatively low voltages obtainable from a magnetoelastic harvester a DC-DC boost based topology with high boosting gain can be considered, [38].

Finally, another feature of a magnetoelastic harvester that challenges the definition and the modeling of the power conversion stage is its strong nonlinearities. For example, these can create on the AC side an additional harmonic content that is not present in the mechanical stimulus and it depends heavily on the harvester operating conditions (e.g. mechanical prestresses and magnetic biases). This pushes to the definition of new circuital multidomain modeling approaches for analyzing the coupling among the mechanical, magnetic and electronic worlds.

\section{Author details}

Daniele Davino, Alessandro Giustiniani, Ciro Visone

University of Sannio - Engineering Department, 82100 Benevento (BN), Italy 


\section{References}

[1] Adly, A.A., Davino, D., Giustiniani, A. \& Visone, C. (2010). Experimental tests of a magnetostrictive energy harvesting device towards its modelling, AIP Journal of Applied Physics, Vol. 107, No. 09A935, May 2010, pp. 1-3.

[2] Anton, S.R. \& Inman D.J. (2008). Vibration energy harvesting for unmanned aerial vehicles, Proceedings of SPIE, Vol. 6928, No. 692824.

[3] Basso, V., Bertotti, G., Serpico, C. \& Visone, C. (1998). Application of an exactly inverse hysteresis model to magnetic field computations, Journal de Physique IV, Vol.8, pp. 639-642.

[4] Bewes, O.G., Thompson, D.J., Jones, C.J.C. \& Wang, A. (2006). Calculation of noise from railway bridges and viaducts Experimental validation of a rapid calculation model, ELSEVIER Journal of Sound and Vibration, Vol. 293, pp. 933-943.

[5] Clark, A.E., Teter, J.P. \& McMasters, O.D. (1988). Magnetostriction "jumps" in twinned $\mathrm{Tb}_{0.3}$ Dy $_{0.7} \mathrm{Fe}_{1.9}$, AIP Journal of Applied Physics, Vol. 63, No. 8, pp. 3910-3912.

[6] Clark, A.E., Wun-Fogle, M., Restorff, J.B. \& Lindberg, J.F. (1992). Magnetomechanical properties of single crystal $\mathrm{Tb}_{x} \mathrm{Dy}_{1-x}$ under compressive stress, IEEE Transactions on Magnetics, Vol. 28, No.5, pp. 3156-3158.

[7] Culler, D. E. \& Hong, W. (2004). Wireless sensor networks, Commun. ACM, Vol.47, pp.30-33.

[8] Davino, D., Giustiniani, A. \& Visone, C. (2009). Capacitive load effects on a magnetostrictive fully coupled energy harvesting device, IEEE Transactions on Magnetics, Vol. 45, No. 10, Oct 2009, pp. 4108-4111.

[9] Davino, D., Giustiniani, A. \& Visone, C. (2009). Analysis of a magnetostrictive power harvesting device with hysteretic characteristics, AIP Journal of Applied Physics, Vol. 105, 07A939.

[10] Davino, D., Giustiniani, A. \& Visone, C. (2011). A two-port nonlinear model for magneto-elastic energy harvesting devices, IEEE Transactions on Industrial Electronics, Vol. 58, No. 6, Jun 2011, pp. 2556-2564.

[11] Davino, D., Giustiniani, A., Visone, C. \& Adly, A.A. (2011). Experimental analysis of vibrations damping due to magnetostrictive based energy harvesting, AIP Journal of Applied Physics, Vol. 109, No. 07E509, Mar 2011, pp. 1-3.

[12] Davino, D., Giustiniani, A. \& Visone, C. (2011). The piezo-magnetic parameters of Terfenol-D: An experimental viewpoint, ELSEVIER Physica B: Condensed Matter, Vol. 407, No. 9, pp. 1427-1432.

[13] Davino, D., Giustiniani, A., Visone, C. \& Adly, A.A. (2011). Experimental analysis of vibrations damping due to magnetostrictive based energy harvesting, AIP Journal of Applied Physics, Vol.109, 07E509.

[14] Davino, D., Giustiniani, A. \& Visone, C. (2011). Effects of hysteresis and eddy currents in magnetostrictive harvesting devices, ELSEVIER Physica B: Condensed Matter, Vol.407, No.9, pp. 1433-1437.

[15] Davino, D., Giustiniani, A., Visone, C. \& Zamboni, W. (2012). Stress-Induced Eddy Currents in Magnetostrictive Energy Harvesting Devices, IEEE Transactions on Magnetics, Vol. 48, No.1, pp. 18-25. 
[16] Dayal, R., Dwari, S. \& Parsa, L. (2011). Design and Implementation of a Direct AC-DC Boost Converter for Low-Voltage Energy Harvesting, IEEE Transactions on Industrial Electronics, Vol. 58, No. 6, pp. 2387-2396.

[17] Del Grosso, A., Inaudi, D. \& Pardi, L. (2002). Overview of european activities in the health monitoring of bridges, Proceedings 1st International Conference on Bridge Maintenance, Safety Management (IABMAS), Barcelona, Spain, pp. 14-17.

[18] Elvin, N.G. \& Elvin A.A., 2011 Vibrational Energy Harvesting From Human Gait, IEEE/ASME Transactions on Mechatronics, DOI: 10.1109/TMECH.2011.2181954, forthcoming.

[19] Ed. Engdahl, G. 2000, Handbook of Giant Magnetostrictive Materials, (Academic, New York).

[20] Galchev, T.V., McCullagh, J., Peterson, R.L. \& Najafi, K. (2011). Harvesting traffic-induced vibrations for structural health monitoring of bridges, IOP Journal of Micromechanics and Microengineering, Vol. 21, No. 104005.

[21] Garibaldi, L., Giorcelli, E. \& Piombo, B.A.D. (1998). ARMAV techniques for traffic excited bridges, ASME Journal of Vibration and Acoustics, Vol. 120, No 3, pp. 713-718.

[22] Hu, J., Xu, F., Huang, A.Q. \& Yuan, F.G. (2011). Optimal design of a vibration-based energy harvester using magnetostrictive material (MsM), IOP Smart Materials and Structures, Vol. 20, No. 015021, Dec 2011, pp. 1-12.

[23] Karami, M.A. \& Inman D.J. (2012). Powering pacemakers from heartbeat vibrations using linear and nonlinear energy harvesters, AIP Applied Physics Letters, Vol. 100, No. 042901, pp. 1-4.

[24] Krejčí, P. (1996). Hysteresis, convexity and dissipation in hyperbolic equations, Gakuto International Series: mathematical sciences and applications, 8, Gakkōtosho, Tokyo.

[25] Lallart, M. \& Guyomar, D. (2011). Nonlinear energy harvesting, IOP Conference Series: Materials Science and Engineering, Vol. 18, 092006, pp. 1-6.

[26] Lee, E.W. (1955). Magnetostriction and magnetomechanical effects, IOP Reports on Progress in Physics, vol. 18, pp. 184-227.

[27] Lee, J.W., Kim, J.D., Yun, C.B., Yi, J.H. \& Shim, J.M. (2002). Health-monitoring method for bridges under ordinary traffic loadings, ELSEVIER Journal of Sound and Vibration, Vol. 257, No. 2, pp. 247-264.

[28] Lesieutre, G.A., Ottman, G.K. \& Hofmann, H.F. (2004). Damping as a Result of Piezoelectric Energy Harvesting, ELSEVIER Journal of Sound and Vibration, Vol. 269, pp. 991-1001.

[29] Liang, J.R. \& Liao W.H. (2009). Piezoelectric Energy Harvesting and Dissipation on Structural Damping, SAGE Journal of Intelligent Material Systems and Structures, Vol. 20, No. 5, pp. 515-527.

[30] Lin, C.W. \& Yang, Y.B. (2005). Use of a passing vehicle to scan the fundamental bridge frequencies: An experimental verification, ELSEVIER Engineering Structures, Vol. 27, pp. 1865-1878.

[31] Lynch, J.P. (2007). An overview of wireless structural health monitoring for civil structures, Philosophical Transactions of the Royal Society A, Vol. 365, pp. 345-372.

[32] Mhetre, M.R., Nagdeo, N.S. \& Abhyankar, H.K. (2011). Micro Energy Harvesting for Biomedical Applications: A Review, Proceedings of 3rd International Conference on Electronics Computer Technology (ICECT), pp. 1-5. 
[33] Mitcheson, P.D., Yeatman, E.M., Rao, G.K., Holmes, A.S. \& Green, T.C. (2008). Energy Harvesting From Human and Machine Motion for Wireless Electronic Devices, Proceedings of IEEE, 96(9), pp. 1457-1486.

[34] Moffett, M.B., Clark, A.E., Wun-Fogle, M., Linberg, J., Teter, J.P. \& McLaughlin, E.A.(1991). Characterization of Terfenol-D for magnetostrictive transducers, Journal of the American Acoustical Society, Vol. 89, No.3, pp. 1448-1455.

[35] Ottman, G.K., Hofmann, H.F., Bhatt, A.C. \& Lesieutre, G.A. (2002). Adaptive piezoelectric energy harvesting circuit for wireless remote power supply, IEEE Transactions on Power Electronics, Vol. 17, No.5, pp. 669-676.

[36] Pereyma, M. (2007). Overview of the Modern State of the Vibration Energy Harvesting Devices, Proceedings of International Conference on Perspective Technologies and Methods in MEMS Design, pp. 107-112.

[37] Restorff, J.B., Wun-Fogle, M. \& Summers, E. (2011). Hysteresis, $d_{33}^{*}$ and $d_{33}$ of $\mathrm{Fe}_{81.6} \mathrm{Ga}_{18.4}$ textured polycrystals, AIP Journal of Applied Physics, Vol. 109, 07A922.

[38] Richelli, A., Comensoli, S. \& Kovacs-Vajna, Z.M. (2012). A DC/DC Boosting Technique and Power Management for Ultralow-Voltage Energy Harvesting Applications, IEEE Transactions on Industrial Electronics, Vol. 59, No. 6, pp. 2701-2708.

[39] Roundy, S. (2005). On the Effectiveness of Vibration-based Energy Harvesting, SAGE Journal of Intelligent Material Systems and Structures, Vol. 16, Oct 2005, pp. 809-823.

[40] Schulze, M.P., Greenough, R.D., \& Galloway, N. (1992). The Stress Dependence of $\mathrm{k}_{33}$, $\mathrm{d}_{33}, \lambda$ and $\mu$ in $\mathrm{Tb}_{0.3} \mathrm{Dy}_{0.7} \mathrm{Fe}_{1.95}$, IEEE Transactions on Magnetics, Vol. 28, No.5, pp. 3159-3161.

[41] Smith, C.A. \& Anderson, E.H. (1995). Passive damping by smart materials: analysis and practical limitations, proceedings of SPIE, 2445, Vol.136, pp. 136-148.

[42] Szarka, G.D., Stark, B.H. \& Burrow, S.G. (2012). Review of Power Conditioning for Kinetic Energy Harvesting Systems, IEEE Transactions on Power Electronics, Vol. 27, No. 2, pp. 803-815.

[43] Turner, J., Cartwright, J., Ha, D.S., Zhang, D. \& Banerjee, S. (2011). On Energy Harvesting Module for Scalable Cognitive Autonomous Nondestructive Sensing Network (SCANSn) System for Bridge Health Monitoring, Proc. of SPIE, 79831T.

[44] Ueno, T. \& Yamada, S. (2011). Performance of Energy Harvester Using Iron-Gallium Alloy in Free Vibration, IEEE Transactions on Magnetics, Vol. 47, No.10, pp. 2407-2409.

[45] Wang, L. \& Yuan, F.G. (2008). Vibration energy harvesting by magnetostrictive material, IOP Smart Materials and Structures, Vol. 17, No. 045009, Jun 2008, pp. 1-14.

[46] Westby, E.R. \& Halvorsen E. (2011). Design and modeling of a patterned-electret-based energy harvester for tire pressure monitoring systems, IEEE/ASME Transactions On Mechatronics, DOI: 10.1109/TMECH.2011.2151203, forthcoming.

[47] Williams, C.B. (1997). Feasibility Study of Vibration-Electric generator for Bridge Vibration sensors, Proceedings Of IMAC-XVI.

[48] Yongping, W., Daining, F., Soh, A.K. \& Hwang, K.C.(2003). Experimental and theoretical study of the nonlinear response of a giant magnetostrictive rod, SPRINGER Acta Mechanica Sinica, Vol. 19, No.4, pp. 324-329.

[49] Yoo, J.H., Pelligrini, G., Datta, S. \& Flatau, A.B. (2011). An examination of Galfenol mechanical magnetic coupling coefficients, IOP Smart Materials and Structures, Vol. 20, No. 075008, Jun 2011, pp. 1-8. 
[50] Zhang, H. (2011). Power generation transducer from magnetostrictive materials, AIP Applied Physics Letters, Vol. 98, No. 232505, Jun 2011, pp. 1-3.

[51] Zhao, X. \& Lord, D.G. (2006). Application of the Villari effect to electric power harvesting, AIP Journal of Applied Physics, Vol. 99, No. 08M703, Apr 2006, pp. 1-3.

[52] Zhu, L. \& Chen, R. (2012). A new synchronized switching harvesting scheme employing current doubler rectifier, ELSEVIER Sensors and Actuators A: Physical, Vol. 174, No. 6, pp. 107-114.

[53] Zucca, M., Bottauscio, O., Beatrice, C. \& Fiorillo, F. (2011). Modeling Amorphous Ribbons in Energy Harvesting Applications, IEEE Transactions on Magnetics, Vol. 47, No.10, pp. 4421-4424.

[54] Zuo, L., Scully, B., Shestani, J. \& Zhou, Y. (2010). Design and characterization of an electromagnetic energy harvester for vehicle suspensions, IOP Smart Materials and Structures, Vol. 19, No. 045003, pp. 1-10. 